\title{
FLAIR* to visualize veins in white matter lesions: A new tool for the diagnosis of multiple sclerosis?
}

\author{
T. Campion ${ }^{1,2}$ (1) - R. J. P. Smith ${ }^{1,3}$ • D. R. Altmann ${ }^{4}$ - G. C. Brito ${ }^{1}$ - B. P. Turner ${ }^{1,2}$ • \\ J. Evanson ${ }^{2}$ I. C. George ${ }^{5,6}$ - P. Sati ${ }^{5}$ - D. S. Reich ${ }^{5}$ - M. E. Miquel ${ }^{2,7} \cdot$ K. Schmierer ${ }^{1,2}$
}

Received: 6 December 2016 / Revised: 22 February 2017 / Accepted: 17 March 2017 /Published online: 13 April 2017

(C) The Author(s) 2017. This article is an open access publication

\begin{abstract}
Objective To explore the potential of a post-processing technique combining FLAIR and $\mathrm{T}_{2} *$ (FLAIR*) to distinguish between lesions caused by multiple sclerosis (MS) from cerebral small vessel disease (SVD) in a clinical setting.

Methods FLAIR and $\mathrm{T}_{2} *$ head datasets acquired at $3 \mathrm{~T}$ of 25 people with relapsing MS (pwRMS) and ten with pwSVD were used. After post-processing, FLAIR* maps were used to determine the proportion of white matter lesions (WML) showing the 'vein in lesion' sign (VIL), a characteristic histopathological feature of MS plaques. Sensitivity and specificity of MS diagnosis were examined on the basis of $>45 \% \mathrm{VIL}^{+}$ and $>60 \% \mathrm{VIL}^{+}$WML, and compared with current dissemination in space (DIS) MRI criteria.

Results All pwRMS had $>45 \% \mathrm{VIL}^{+}$WML (range $58-100 \%$ ) whilst in pwSVD the proportion of $\mathrm{VIL}^{+}$WML was
\end{abstract}

T. Campion

thomascampion@nhs.net

1 Blizard Institute (Neuroscience), Queen Mary University of London, London, UK

2 Barts Health NHS Trust, Emergency Care and Acute Medicine Clinical Academic Group Neuroscience, The Royal London Hospital, Whitechapel Road, London, UK

3 Gloucestershire Hospitals NHS Trust, Cheltenham, UK

4 Department of Medical Statistics, London School of Hygiene and Tropical Medicine, London, UK

5 Translational Neuroradiology Section, National Institute of Neurological Disorders and Stroke, NIH, Bethesda, MD, USA

6 Department of Neurology, Yale School of Medicine, New Haven, CT, USA

7 William Harvey Research Institute (Cardiovascular Biomedical Research Unit), Queen Mary University of London, London, UK significantly lower (0-64\%; mean $32 \pm 20 \%)$. Sensitivity based on $>45 \% \mathrm{VIL}^{+}$was $100 \%$ and specificity $80 \%$ whilst with $>60 \% \mathrm{VIL}^{+}$as the criterion, sensitivity was $96 \%$ and specificity $90 \%$. DIS criteria had $96 \%$ sensitivity and $40 \%$ specificity. Conclusion FLAIR* enables $\mathrm{VIL}^{+}$WML detection in a clinical setting, facilitating differentiation of MS from SVD based on brain MRI.

Key points

- FLAIR* in a clinical setting allows visualization of veins in white matter lesions.

- Significant proportions of MS lesions demonstrate a vein in lesion on MRI.

- Microangiopathic lesions demonstrate a lower proportion of intralesional veins than MS lesions.

- Intralesional vein-based criteria may complement current MRI criteria for MS diagnosis.

Keywords Multiple sclerosis · MRI · White matter · Neuroimaging $\cdot$ Central vein

\section{Introduction}

No noninvasive test result is fully specific for the diagnosis of multiple sclerosis (MS), one of the most common conditions causing chronic neurological disability. The current diagnostic criteria for MS ('McDonald' criteria) are based on clinical and paraclinical evidence, including magnetic resonance imaging (MRI), of dissemination in time (DIT) and space (DIS) of lesions suggestive of inflammatory demyelination. These criteria also rely on the exclusion of alternative conditions that would better explain a patient's symptoms, signs and results of investigations [1]. The stipulation of 'no better explanation' underpins the character of MS as a diagnosis of exclusion. 
MRI of the brain and spinal cord is the single most important investigation in the diagnostic work-up of people suspected of having MS, and serves both the diagnostic criteria laid down by the International Panel [1] and the exclusion of differential diagnoses. However, lesions suggestive of demyelination detected on conventional $\mathrm{T}_{2^{-}}$ weighted MRI may in fact have a different pathological substrate, such as cerebral small vessel disease (SVD), migraine or infections [2]. The probability of one of these alternative diagnoses may depend, for example, on age, vascular risk factors or genetic background. Strict adherence to the current criteria can therefore delay the definitive diagnosis of MS and, as a result, disease-modifying treatment (DMT). Given the evidence that treatment of people with MS, particularly those with a relapsing course (pwRMS), is most effective when started early, such delay may be clinically important [3-5].

Another limitation of current MRI techniques is apparent in people with MS who also have risk factors for SVD. Comorbidity may compromise correct allocation of new lesions to their cause [6], and such uncertainty may directly impact on treatment decisions $[7,8]$.

In order to further improve MRI as a tool to support a diagnosis of MS, one of its characteristic histological features has recently been revisited: the vein about which MS lesions almost invariably evolve $[9,10]$. This perivenous morphology of MS lesions appears to have become accessible in vivo using MRI techniques susceptible to iron in deoxygenated haemoglobin, such as susceptibility or $\mathrm{T}_{2}{ }^{*}$ weighted imaging [11].

In a study using $\mathrm{T}_{2}{ }^{*}$-weighted imaging at $7 \mathrm{~T}$, the detection of 'veins in lesions' (VIL) suggested VIL may be useful as a diagnostic marker for MS [12]. A further study by the same group suggested a proportion of $40 \%$ or more VIL positive $\left(\mathrm{VIL}^{+}\right)$white matter lesions (WML) distinguished people with MS (pwMS) from people with WML of a different aetiology with $100 \%$ positive and negative predictive values [13].

Whilst 7T MRI currently provides the best platform in terms of signal-to-noise and resolution to detect WML and cerebral veins alike, 7T scanners are not widely available, particularly in clinical settings, and a substantial number of WML and cerebral veins can also be visualized at 3T [14]. At $3 \mathrm{~T}$, a proportion of $45 \% \mathrm{VIL}^{+} \mathrm{WML}$ has been shown to correctly categorize patients as having MS or SVD lesions [15].

In the study reported here, $\mathrm{T}_{2}{ }^{*}$-weighted $3 \mathrm{D}$ echo-planarimaging (3D EPI), to detect VIL, and $\mathrm{T}_{2}$-weighted fluid attenuated inversion recovery (FLAIR) sequences, to detect WML, were acquired at $3 \mathrm{~T}$ and combined to generate FLAIR* images, first described by Sati and co-workers [16].

Using datasets acquired in a routine clinical setting we explored the sensitivity and specificity of a set of criteria based on FLAIR* for comparison with the revised International Panel MRI criteria currently used to support a diagnosis of MS. We further compared our results to a group of patients with WML and a clinical profile consistent with SVD to determine whether the proportion of $\mathrm{VIL}^{+} \mathrm{WML}$ could be useful in differentiating MS from SVD.

\section{Materials and methods}

\section{Subjects}

This study was approved by the National Research Ethics Committee North West - Haydock (15/NW/0065) and undertaken at a single centre, The Royal London Hospital (RLH), Barts Health NHS Trust, London, UK. Datasets were used of patients who had undergone MRI as part of routine care. Written informed consent was obtained prior to any study procedure. Principal sources of referrals were the Neurology and Neuroinflammation services of the RLH, with additional referrals facilitated by the National Institute of Health Research (NIHR), North Thames Clinical Research Network.

For inclusion in the study MRI brain studies had to show at least one white matter lesion. Datasets of pwRMS were included if the diagnosis was confirmed according to the most recent International Panel criteria [1]. Datasets of people with SVD (pwSVD) were included if they (i) did not have a clinical diagnosis of MS (and were not suspected of having MS) and (ii) had at least two of six risk factors for SVD (diabetes, high blood pressure, smoking, hypercholesterolaemia, ischaemic heart disease, peripheral vascular disease [17]). Datasets of patients with any additional CNS pathology were excluded.

\section{Data acquisition}

All images were acquired on a Philips Achieva 3T TX system (Philips Healthcare, Best, The Netherlands) based at St Barts Hospital of Barts Health NHS Trust using either a 16element neurovascular coil or an 8-element head coil. The protocol included a $\mathrm{T}_{2}{ }^{*}$-weighted $3 \mathrm{D}$ segmented EPI sequence (TE $29 \mathrm{~ms}$, TR $53 \mathrm{~ms}$, flip angle $10^{\circ}$, EPI factor 15 , field of view $240 \times 240 \times 180 \mathrm{~mm}^{3}, 0.55 \times 0.55 \times 0.55$ $\mathrm{mm}^{3}$ resolution, SENSE acceleration $2 \times 2$, total acquisition time $3 \mathrm{~min} 50 \mathrm{~s}$ ) [18] and a 3D FLAIR sequence (VISTA protocol, TE $372 \mathrm{~ms}$, TR $4800 \mathrm{~ms}$, TI $1600 \mathrm{~ms}$, field of view $240 \times 240 \times 180 \mathrm{~mm}^{3}, 1 \times 1 \times 1 \mathrm{~mm}^{3}$ resolution, SENSE acceleration $2 \times 2.6$, total acquisition time $6 \mathrm{~min}$ ) after injection of $10 \mathrm{ml}$ of $0.5 \mathrm{mmol} / \mathrm{ml}$ gadoteric acid contrast agent. Contrast was injected manually right before the $\mathrm{T}_{2} *$ sequence was acquired. FLAIR was acquired $13 \mathrm{~min}$ post contrast administration. The scanner manufacturer 
provided both sequences. 3D $\mathrm{T}_{1}$ (fast field echo; before and after administration of gadolinium) and $3 \mathrm{D} \mathrm{T}_{2}$ (turbo spin echo) sequences were also obtained in order to assess lesions according to the McDonald criteria. Due to our local scanning protocols, these scanning parameters differ slightly from those described by Sati et al. [16]; a standard contrast dose with manual injection was used instead of a weight-adjusted contrast dose via power injector, and thus the delay to the FLAIR sequence was longer.

\section{Image processing}

FLAIR * images were constructed using the FLAIR and $\mathrm{T}_{2} *$ datasets using MIPAV (mipav.cit.nih.gov) and JIST (www. nitrc.org/projects/jist/) image processing software. Postprocessing was conducted using a processing pipeline as described previously [16]. All images were first reformatted to the axial orientation without interpolation. To correct for motion between acquisitions, the FLAIR dataset was coregistered to the 3D EPI sequence using a rigid registration with six degrees of freedom, normalised mutual information as the cost function, and windowed sinc interpolation. The registered FLAIR images were then interpolated to the same spatial resolution and multiplied to the 3D EPI sequence to obtain the FLAIR* images (Fig. 1).

\section{Data analysis}

MRI data were assessed by a neuroradiology fellow (RJPS) and a radiology trainee with specific neuroradiology training (TC), supervised by a senior consultant neuroradiologist (JE). Both assessors were blinded to clinical information. WML were defined as discrete areas of high signal intensity on FLAIR* images with a minimum diameter of $3 \mathrm{~mm}$. Their number, location and whether or not they were $\mathrm{VIL}^{+}$(defined as containing a hypo-intense line or dot on axial FLAIR*) were recorded (Fig. 2). Inter-observer agreement of the presence/absence of $\mathrm{VIL}^{+} \mathrm{WML}$ was assessed on a lesion level using Cohen'skcoefficient calculated using Microsoft Excel. Additional statistical analysis was performed using StatPlus.

Only WML identified by both observers were included in the analysis. Where there was disagreement between observers about the presence of VIL, images were jointly reevaluated and a consensus reached. The proportion of $\mathrm{VIL}^{+}$ WML was then determined for each patient. A proportion of $45 \%$ or more of $\mathrm{VIL}^{+}$WML was considered diagnostic for MS (the 'VIL45' criterion) [15]. The proportion in the MS and SVD cohorts was compared using the Mann Whitney test. We also assessed the value of a higher threshold (a proportion of $60 \%$ or more; 'VIL60' criterion) to determine whether this would impact on sensitivity and specificity.

Recent evidence suggests that a thorough assessment of only a limited number of 'morphologically characteristic lesions' (MCLs, i.e. VIL ${ }^{+}$WML) can be used [15] as an alternative to calculating the relative proportion of $\mathrm{VIL}^{+}$ WML. We applied a slightly simplified version of these criteria ('rule of six criteria') to our dataset as follows:

1. If there were six or more $\mathrm{VIL}^{+} \mathrm{WML}$, a diagnosis of MS was assigned.

2. If there were fewer than six $\mathrm{VIL}^{+} \mathrm{WML}$, but $\mathrm{VIL}^{+} \mathrm{WML}$ outnumbered $\mathrm{VIL}^{-}$WML, a diagnosis of MS was assigned.

3. If neither of these conditions was met, MRI was deemed not confirmatory for a diagnosis of MS.

Finally, datasets of each participant were inspected to determine whether they fulfill the standard DIS and DIT criteria. DIS was considered fulfilled if lesions were identified on $\mathrm{T}_{2}$-weighted scans in two or more areas characteristic for MS. DIT was considered fulfilled if both enhancing and non-enhancing WML were present in parallel on the 3D $\mathrm{T}_{1}$ post-gadolinium sequence [1].

Sensitivity, specificity and accuracy for the diagnosis of MS were calculated for three VIL-based criteria (VIL45, VIL60 and 'rule of six') and two McDonald-based criteria
Fig. 1 Construction of FLAIR* images. (a) FLAIR, axial slice. (b) $\mathrm{T}_{2} *$, axial slice. (c) Combined images create FLAIR*, axial slice. White arrow indicates intralesional vein
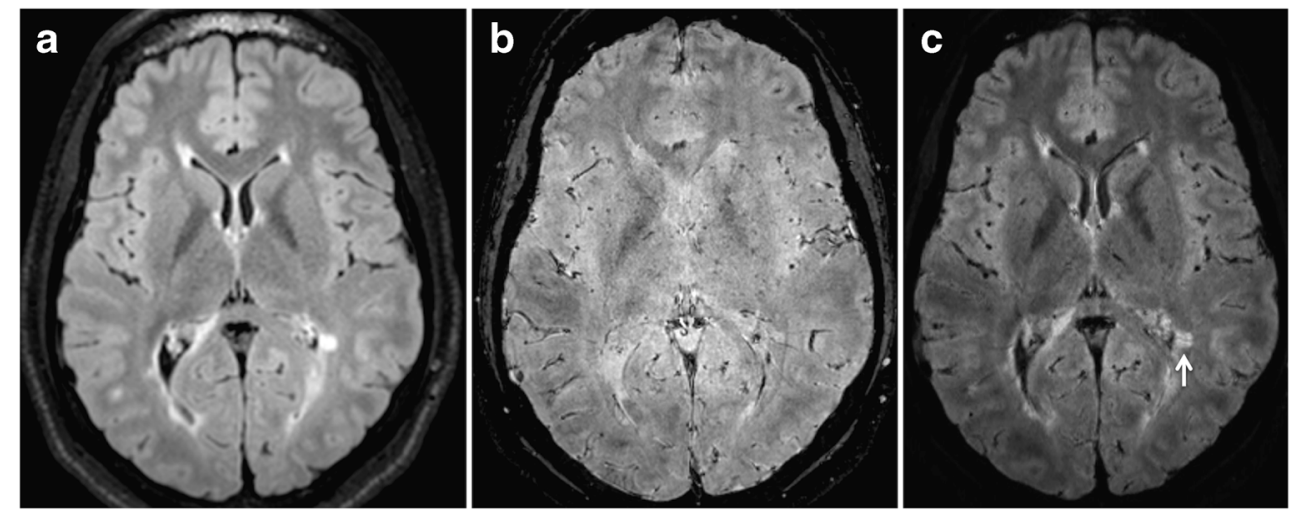


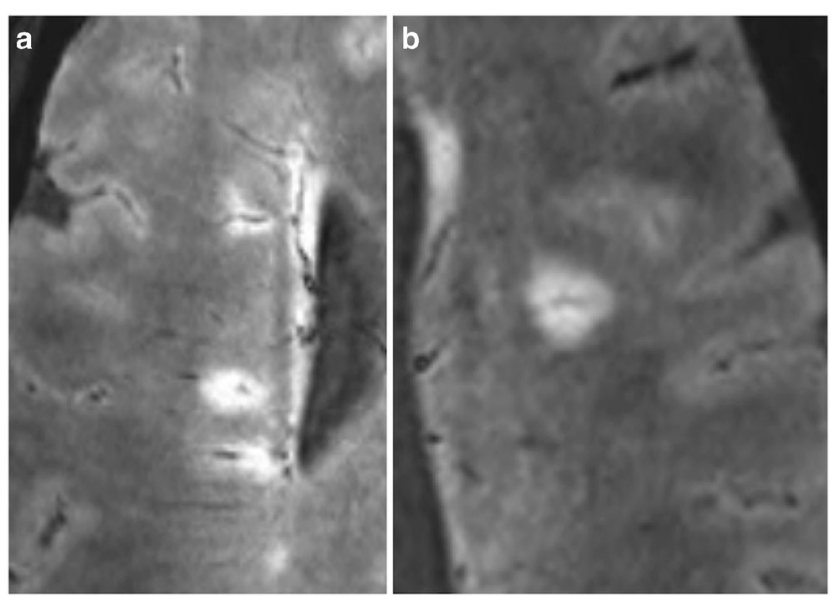

Fig. $2 \mathrm{VIL}^{+}$lesions on FLAIR* in a patient with multiple sclerosis. (a) Multiple periventricular VIL+ lesions. (b) Subcortical VIL+ lesion. VIL vein in lesion

(DIS alone, and DIS and DIT). The McNemar test was used to compare the number of pwRMS and pwSVD who were $\mathrm{VIL}^{+} 5^{+}$and VIL60 ${ }^{+}$with the number of pwRMS who fulfilled the DIS, and DIS and DIT (McDonald 2010 MRI) criteria.

\section{Results}

Datasets of 25 pwRMS (14 men and 11 women; age $41 \pm 11$ years; disease duration $6 \pm 5$ years) and 10 pwSVDs (four men and six women; age $59 \pm 9$ years) were used. pwSVDs were older $(p<0.01)$. Fourteen of 25 pwRMS were on various DMT at the time of scanning.

The inter-observer agreement for determining whether a given WML was $\mathrm{VIL}^{+}$, calculated on a lesion by lesion basis, was moderate $(K=0.70)[19]$.

In pwRMS, a total of $338 \mathrm{WML}$ were identified (range 5-31; mean $14 \pm 7$ ). Of these, $291 \mathrm{WML}(86 \%)$ were $\mathrm{VIL}^{+}$. At least $58 \% \mathrm{VIL}^{+}$WML were detected in each pwRMS

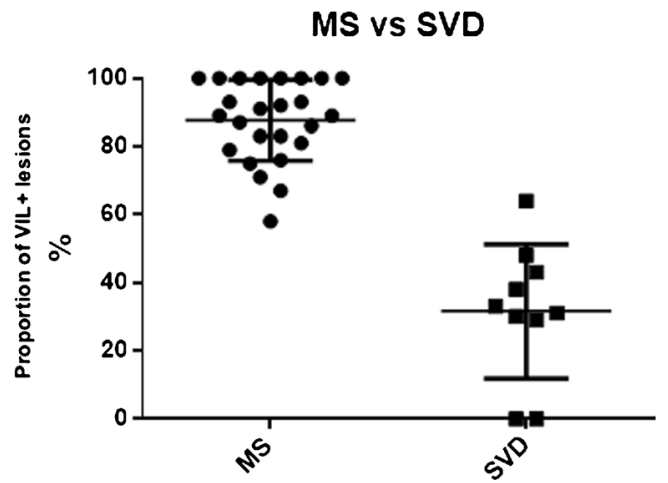

Fig. 3 Proportion of VIL ${ }^{+}$lesions in multiple sclerosis (MS) and cerebral small vessel disease (SVD) cohorts. VIL vein in lesion (range $58-100 \%$; mean $88 \pm 12 \%$ ). All pwRMS met the 'rule of six' criteria for MS.

Twenty-four pwRMS (96\%) fulfilled the McDonald DIS criterion, whilst three (12\%) fulfilled both DIS and DIT.

There was a significant difference in the mean proportion of $\mathrm{VIL}^{+}$WML between pwRMS on DMT and patients who were not (on DMT 83\%, not on DMT 94\%, p = 0.013).

In pwSVD, a total of $136 \mathrm{WML}$ was identified (range 0 33 ; mean $13 \pm 9)$. Of these, $54(40 \%)$ were $\mathrm{VIL}^{+}$. The mean proportion of $\mathrm{VIL}^{+} \mathrm{WML}$ was significantly lower (range 0 $64 \%$; mean $32 \pm 20 \%)$ than in pwRMS (p<0.0001) (Fig. 3). Three of ten pwSVDs met the 'rule of six' criteria for MS. Six of ten pwSVDs fulfilled the McDonald 2010 DIS criteria; none fulfilled both DIS and DIT.

The VIL45 criterion had a diagnostic sensitivity of $100 \%$ and a specificity of $80 \%$. The respective figures for VIL60 were $96 \%$ and $90 \%$. The 'rule of six' criterion had a sensitivity of $100 \%$ and a specificity of $70 \%$.

The McDonald 2010 DIS criterion had a sensitivity of $96 \%$ and a specificity of $40 \%$ (Table 1 ).

\section{Discussion}

The McDonald MRI criteria are based on a DIS component, which depends on the morphology and distribution pattern of WML, and a DIT component inferred from either (i) the presence in parallel of Gadolinium-enhancing $\left(\mathrm{Gd}^{+}\right)$and non-enhancing $\left(\mathrm{Gd}^{-}\right) \mathrm{WML}$ at baseline, or (ii) new WML, be they $\mathrm{Gd}^{+}$or $\mathrm{Gd}^{-}$, on follow-up MRI. Although the most recent edition of these criteria has improved and simplified the interpretation of MRI scans to support a diagnosis of MS [1], their applicability in clinical practice remains imperfect $[20,21]$.

As an alternative to, or perhaps to complement, the pattern-based approach used in the McDonald MRI criteria, the detection and interpretation of $\mathrm{VIL}^{+} \mathrm{WML}$ in vivo benefits from the routine availability of MRI techniques susceptible to deoxygenated blood [22], which appears to enable the visualisation of a histological hallmark of MS WML described for over 160 years, the perivenular morphology of WML [23]. Although we are not aware of any correlative post mortem MRI/pathology studies, the topography of hypo-intensity within WML and the presence of deoxyhaemoglobin within veins (which increase susceptibility effects) suggest $\mathrm{VIL}^{+}$WML most likely represent lesions that have emerged around veins.

Using manufacturer-provided sequences acquired at a routine clinical field strength, FLAIR* detected VIL in over $60 \%$ of WML in all but one pwRMS included in this study. At a single time point, and using either the threshold of $45 \%$ as proposed in earlier studies using 7T [12] and 3T [15] MRI, or a threshold of $60 \%$, the presence of $\mathrm{VIL}^{+} \mathrm{WML}$ 
Table 1 Comparison of different criteria for diagnosis of multiple sclerosis (MS)

\begin{tabular}{|c|c|c|c|c|c|c|}
\hline & & & & $\begin{array}{l}\text { Sensitivity } \\
(\%) \\
(95 \% \mathrm{CI})\end{array}$ & $\begin{array}{l}\text { Specificity } \\
(\%) \\
(95 \% \mathrm{CI})\end{array}$ & $\begin{array}{l}\text { Accuracy } \\
(\%) \\
(95 \% \mathrm{CI})\end{array}$ \\
\hline \multirow{2}{*}{$\begin{array}{l}\text { McDonalds DIS } \\
\text { Criterion }\end{array}$} & & & & 96 & 40 & 80 \\
\hline & Positive & Negative & Total & $(80-100)$ & $(12-74)$ & $(63-92)$ \\
\hline MS & 24 & 1 & 25 & & & \\
\hline SVD & 6 & 4 & 10 & & & \\
\hline Total & 31 & 4 & & & & \\
\hline \multirow[t]{2}{*}{$\mathrm{VIL}+45 \%$} & & & & 100 & 80 & 94 \\
\hline & Positive & Negative & Total & $(86-100)$ & (44-97) & $(81-99)$ \\
\hline MS & 25 & 0 & 25 & & & \\
\hline SVD & 2 & 8 & 10 & & & \\
\hline Total & 27 & 8 & & & & \\
\hline \multirow[t]{2}{*}{$\mathrm{VIL}+60 \%$} & & & & 96 & 90 & 94 \\
\hline & Positive & Negative & Total & $(80-100)$ & $(56-100)$ & $(81-99)$ \\
\hline MS & 24 & 1 & 25 & & & \\
\hline SVD & 1 & 9 & 10 & & & \\
\hline Total & 25 & 10 & & & & \\
\hline \multirow[t]{2}{*}{ Rule of Six } & & & & 100 & 70 & 91 \\
\hline & Positive & Negative & Total & $(86-100)$ & (35-93) & (77-98) \\
\hline MS & 25 & 0 & 25 & & & \\
\hline SVD & 3 & 7 & 10 & & & \\
\hline Total & 28 & 7 & & & & \\
\hline
\end{tabular}

$C I$ confidence interval, $S V D$ small vessel disease, $D I S$ dissemination in space, $s$ vein in lesion corroborated the diagnosis of MS in all (bar one in the VIL60 analysis) participants studied, as did the rule of six, though with a lower specificity.

The McDonald DIS criterion was as sensitive as the VILbased indices employed; however, its specificity was significantly lower (40\% vs. $70-90 \%$ ). Whilst $40 \%$ appears particularly low, it has previously been shown that DIT information is a key contributor towards specificity in the McDonald MRI criteria [24]. Our study supports previous work in demonstrating a significantly higher proportion of $\mathrm{VIL}^{+}$WML in pwMS compared to pwSVD [12].

However, given that two pwSVDs were VIL $45^{+}$and one VIL $60^{+}$, the thresholds used were not absolute discriminators. The reason for this may be that veins can incidentally cross SVD lesions thereby giving the wrong impression of a 'classic' $\mathrm{VIL}^{+}$WML. Moreover, the lack of post mortem evidence leaves some uncertainty about what exact proportion of hypointensities in WML indeed represent veins. On the other hand, $\mathrm{VIL}^{+}$WML in pwMS may be missed due to their small size/diameter. To maximize diagnostic value it may therefore be necessary to combine criteria largely based on lesion morphology with criteria based on distribution pattern and location. A more stringent definition of what constitutes an MS related VIL may also likely be required [10].

The difference in the mean proportion of $\mathrm{VIL}^{+}$WML supports the growing body of evidence suggesting this radiological sign is a useful additional discriminator, in contrast to previous research demonstrating no added benefit [25]. Susceptibility weighted imaging to demonstrate $\mathrm{VIL}^{+} \mathrm{WML}$ also suggested high sensitivity and specificity for the diagnosis of MS $[26,27]$. However, inspection of the FLAIR* maps acquired in our and previous studies [16, 28] suggests there are advantages in combining high isotropic resolution $\mathrm{T}_{2}{ }^{*}$ weighted MRI with a well-established technique for WML detection: recognizing $\mathrm{VIL}^{+}$WML on FLAIR* appears more straightforward than on $\mathrm{T}_{2}{ }^{*}$ alone (Fig. 4). This is in line with another recent study demonstrating that using FLAIR and FLAIR* as part of a global assessment of whether $>40 \%$ WML contain a vein improves diagnostic accuracy for MS without the need to assess every single lesion [28].

\section{Limitations}

Though image reviewers were often unaware of the diagnosis, systematic blinding was not undertaken. Moreover, most pwRMS were on DMT when MRI datasets were acquired, compromising the potential to reveal $\mathrm{Gd}^{+} \mathrm{WML}$ and likely explaining the particularly small number (3) of pwRMS in our study meeting DIT criteria at a single time point [24]. A limitation inherent in the method is that identification of $\mathrm{VIL}^{+}$ WML remains to some extent subjective; although agreement in our study between raters was moderate $(\kappa=0.70)$, specific 
Fig. $4 \mathrm{~T}_{2}$ * versus FLAIR*. (a-c) Partial $\mathrm{T}_{2} *$ slices from three patients with multiple sclerosis (MS): (a) axial, (b) axial and (c) coronal. White circle indicates vein, although lesions not clearly identified. (d-f) Corresponding partial FLAIR* slices at same level. White circle indicates white matter lesion around the same vein
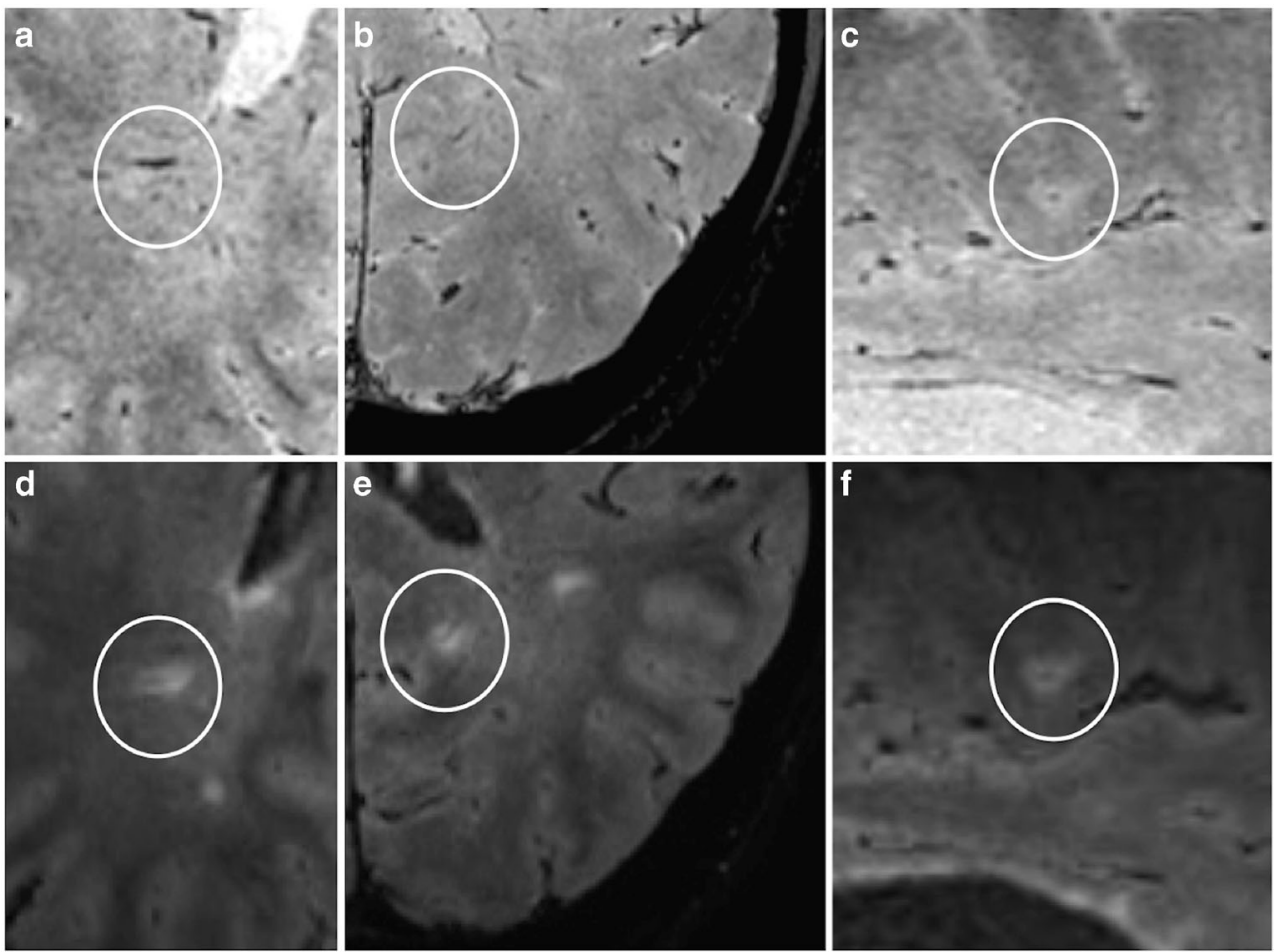

training and reading criteria may be required to introduce the VIL sign as a standard in clinical practice.

The acquisition protocol of our study was slightly different from previous studies using similar methodology [16, 28]. In particular, manual administration of the contrast medium followed by the $\mathrm{T}_{2}$ * sequence, and a different dosing regime (standard instead of weight-adjusted dose) may have altered the vein versus lesion contrast with a possible impact on the VIL45 and VIL60 indices and inter-rater agreement.

Finally, pwSVD were older than those in the MS group, thus providing an imperfect match for the pwRMS cohort. However, higher numbers of WML are to be expected with age, and as such older people should represent a more challenging comparator group to test the VIL criteria. Future studies should compare FLAIR* with McDonald criteria in a larger cohort of people where MS is the suspected diagnosis, i.e. people with a first manifestation of symptoms and/or signs suggestive of demyelination, and acquire FLAIR* at the time of presentation and after defined subsequent intervals. FLAIR* has already shown promise in differentiating between MS and migraine, for example [29].

\section{Conclusion}

In a clinical setting, and using standard manufacturer-supplied sequences, FLAIR* at 3 T reliably enabled in vivo detection of $\mathrm{VIL}^{+}$WML, which resemble a characteristic histological feature of MS. All pwRMS were VIL45 $5^{+}$, underpinning previous data acquired in a research setting. The mean proportion of $\mathrm{VIL}^{+}$WML was significantly higher in pwRMS compared to pwSVD.

Our data suggest that using either VIL45 or VIL60 is as sensitive, and potentially more specific, for the diagnosis of MS than current McDonald 2010 MRI criteria. FLAIR* may thus support the development of more accurate and easy to use MRI criteria for a diagnosis of MS. In line with the recent first consensus statement on the evaluation of central veins in WML [10], further prospective and comparative studies are required to confirm the diagnostic value of VIL, and the techniques used to visualize these and other features of MS.

Acknowledgements We thank the Royal College of Radiologists for funding this study (pump priming grant to RJPS). MEM is partly funded (20\%) by the Barts and the London National Institute for Health Research Cardiovascular Biomedical Research Unit. Additional study support (ICG, PS, DSR) was provided by the Intramural Research Program of the National Institute of Neurological Disorders and Stroke, USA. KS has been supported by a Higher Education Funding Council for England Clinical Senior Lectureship. Daniele Carassiti kindly prepared Fig. 3.

\section{Compliance with ethical standards}

Guarantor The scientific guarantor of this publication is Dr Klaus Schmierer.

Conflict of interest The authors of this manuscript declare relationships with the following companies:

Dr Klaus Schmierer is a principal investigator of trials sponsored by Medday, Novartis, Roche and Teva. He has received speaking honoraria 
from, and served on advisory boards for, Biogen, Novartis, Teva, MerckSerono and Merck Inc; and received research support from Novartis. Dr Ben Turner is a principal investigator of clinical trials for Biogen, SanofiAventis, Genzyme and Roche and received travel grants from these companies. All other authors have no relationships/disclosures/conflicts of interest.

Funding This study has received funding from the Royal College of Radiology (Pump Priming Grant to RJPS). KS has been supported by a Higher Education Funding Council for England Clinical Senior Lectureship. MEM is partly funded by the Barts and the London National Institute for Health Research Cardiovascular Biomedical Research Unit. Additional study support (ICG, PS, DSR) was provided by the Intramural Research Program of the National Institute of Neurological Disorders and Stroke, USA.

Statistics and biometry Dr Altmann is a senior biostatistician and provided the statistical support for this study.

Informed consent Written informed consent was obtained from all subjects (patients) in this study.

Ethical approval Institutional Review Board approval was obtained.

\author{
Methodology \\ - Retrospective \\ - Experimental \\ - Performed at one institution
}

Open Access This article is distributed under the terms of the Creative Commons Attribution 4.0 International License (http:// creativecommons.org/licenses/by/4.0/), which permits unrestricted use, distribution, and reproduction in any medium, provided you give appropriate credit to the original author(s) and the source, provide a link to the Creative Commons license, and indicate if changes were made.

\section{References}

1. Polman CH, Reingold SC, Banwell B et al (2011) Diagnostic criteria for multiple sclerosis: 2010 revisions to the McDonald criteria. Ann Neurol 69:292-302

2. Charil A, Yousry TA, Rovaris M et al (2006) MRI and the diagnosis of multiple sclerosis: expanding the concept of "no better explanation". Lancet Neurol 5:841-852

3. Kappos L, Freedman MS, Polman CH et al (2007) Effect of early versus delayed interferon beta- $1 \mathrm{~b}$ treatment on disability after a first clinical event suggestive of multiple sclerosis: a 3-year follow-up analysis of the BENEFIT study. Lancet 370:389-397

4. Coles AJ, Cox A, Le Page E et al (2006) The window of therapeutic opportunity in multiple sclerosis: evidence from monocolonal antibody therapy. J Neurol 253:98-108

5. Leist TP, Comi G, Cree BA et al (2014) Effect of oral cladribine on time to conversion to clinically definite multiple sclerosis in patients with a first demyelinating event (ORACLE MS): a phase 3 randomised trial. Lancet Neurol 13:257-267

6. Geraldes R, Esiri MM, Deluca GC et al. (2016) Age-related small vessel disease: a potential contributor to neurodegeneration in multiple sclerosis. Brain Pathology

7. Giovannoni G, Turner B, Gnanapavan S, Offiah C, Schmierer K, Marta $M$ (2015) Is it time to target no evident disease activity (NEDA) in multiple sclerosis? Multiple Sclerosis Relat Disord 4:329-333
8. Natalizumab for the treatment of adults with highly active relapsing-remitting multiple sclerosis. NICE technology appraisal guideline [TA127]. Available at: http://www.nice.org.uk/guidance/ TA127/chapter/1-guidance. Accessed on: June 2015

9. Lassmann H (2005) Multiple sclerosis pathology: evolution of pathogenetic concepts. Brain Pathol 15:217-222

10. Sati P, Jiwon O, Todd Constable R, et al. (2016) The central vein sign and its clinical evaluation for the diagnosis of multiple sclerosis: a consensus statement from the North American Imaging in Multiple Sclerosis Collective. Nat Rev Neurol

11. Tan IL, van Schijndel RA, Pouwels PJ et al (2000) MR venography of multiple sclerosis. AJNR 21:1039-1042

12. Mistry N, Dixon J, Tallantyre E et al (2013) Central veins in brain lesions visualized with high-field magnetic resonance imaging. JAMA 70:623-628

13. Tallantyre EC, Dixon JE, Donaldson I et al (2011) Ultra-high-field imaging distinguishes MS lesions from asymptomatic white matter lesions. Neurology 76:534-539

14. Tallantyre EC, Morgan PS, Dixon JE et al (2009) A comparison of $3 \mathrm{~T}$ and $7 \mathrm{~T}$ in the detection of small parenchymal veins within MS lesions. Invest Radiol 44:491-494

15. Mistry N, Abdel-Fahim R, Samaraweera A et al (2015) Imaging central veins in brain lesions with 3-T T2*-weighted magnetic resonance imaging differentiates multiple sclerosis from microangiopathic brain lesions. Mult Scler. doi:10.1177/1352458515616700

16. Sati P, George IC, Shea CD, Gaitan MI, Reich DS (2012) FLAIR*: a combined MR contrast technique for visualizing white matter lesions and parenchymal veins. Radiology 265:926-932

17. Staals J, Makin SDJ, Doubal FN et al (2014) Stroke subtype, vascular risk factors, and total MRI brain small-vessel disease burden. Neurology 83:1228-1234

18. Sati P, Thomasson DM, Lin N et al (2014) Rapid, high-resolution, whole-brain, susceptibility-based MRI of multiple sclerosis. Mult Scler 11:1464-1470

19. McHugh ML (2012) Interrater reliability: the kappa statistic. Biochem Med (Zagreb) 22:276-282

20. Hawkes CH, Giovannoni G (2010) The McDonald criteria for multiple sclerosis: time for clarification. Mult Scler 16:566-575

21. Schmierer K, Turner BP (2012) The second revision of 'McDonald' criteria for the diagnosis of multiple sclerosis. ACNR 12:3-4

22. Mittal S, Wu Z, Neelavalli J, Haacke EM (2009) Susceptibilityweighted imaging: technical aspects and clinical applications, part 2. AJNR 30:232-252

23. Charcot J-M (1868) Histologie de la sclérose en plaques. La Lancette Française, Gazette des Hôpitaux Civils et Militaires 41:554-555

24. Swanton JK, Rovira A, Tintore M et al (2007) MRI criteria for multiple sclerosis in patients presenting with clinically isolated syndromes: a multicentre retrospective study. Lancet Neurol 6:677-686

25. Lummel N, Boeckh-Behrens T, Schoepf V, Burke M, Bruckmann H, Lin J (2011) Presence of a central vein within white matter lesions on susceptibility weighted imaging: a specific finding for multiple sclerosis? Neuroradiology 53:311-317

26. Lane JI, Bolster B, Campeau NG, Welker KM, Gilbertson JR (2015) Characterization of multiple sclerosis plaques using susceptibilityweighted imaging at 1.5T: can perivenular localization improve specificity of imaging criteria? J Comput Assist Tomogr 39:317-320

27. Kilsdonk ID, Wattjes MP, Lopez-Soriano A et al (2014) Improved differentiation between MS and vascular brain lesions using FLAIR* at 7 Tesla. Eur Radiol 24:841-849

28. George IC, Sati P, Absinta M et al (2016) Clinial 3-tesla FLAIR* MRI improves diagnostic accuracy in multiple sclerosis. Mult Scler 22:1578-1586

29. Solomon AJ, Schindler MK, Howard DB et al (2015) "Central vessel sign" on 3T FLAIR* MRI for the differentiation of multiple sclerosis from migraine. Ann Clin Transl Neurol 3:82-87 